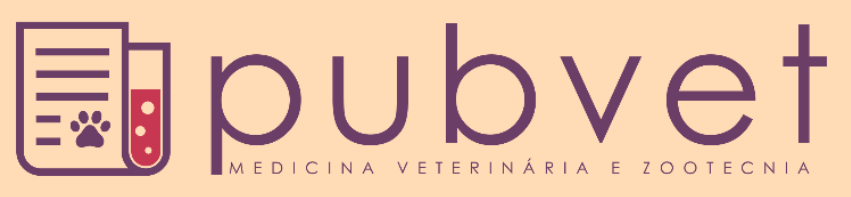

https://doi.org/10.31533/pubvet.v13n8a396.1-6

\title{
Desvio portossistêmico congênito em cães: Revisão
}

\author{
Jéssica Francielle Camargo ${ }^{1 *} \bullet$, Bruna Santos dos $\operatorname{Santos}^{1 \bullet}$, Moisés Westenhofen ${ }^{2} \bullet$, Julia \\ Birnie Farias $^{3}{ }^{\circ}$, Bibiana Lopes $^{4}{ }^{\circ}$, Alexandre Mazzanti ${ }^{5}{ }^{\circ}$, Emerson Antonio Contesini ${ }^{6}{ }^{\circ}$ \\ ${ }^{I}$ Mestranda no Programa de Pós-Graduação em Ciências Veterinárias, Universidade Federal do Rio Grande do Sul. Porto Alegre-RS. Brasil. \\ ${ }^{2}$ Médico Veterinário, Brasil. \\ ${ }^{3}$ Residente em saúde coletiva, Universidade Federal do Pampa. Alegrete-RS. Brasil. \\ ${ }^{4}$ Residente em diagnóstico por imagem, Universidade Federal de Santa Maria. Santa Maria-RS. Brasil. \\ ${ }^{5}$ Professor da Universidade Federal de Santa Maria, curso de Medicina Veterinária. Santa Maria-RS. Brasil. \\ ${ }^{6}$ Professor da Universidade Federal do Rio Grande do Sul, Faculdade de Medicina Veterinária. Porto Alegre-RS. Brasil. \\ *Autor para correspondência, e-mail: jessicavetcamargo@gmail.com
}

Resumo. O desvio portossistêmico ou shunt portossistêmico é uma afecção comumente vista em cães. Essa doença se trata de uma anomalia vascular que faz comunicação entre a circulação sistêmica e a circulação portal. Devido à essa comunicação vascular anômala, o sangue portal proveniente da drenagem de órgãos como estômago, intestino, pâncreas e baço, passa diretamente para a circulação sistêmica, desviando do fígado que é responsável pela desintoxicação de diversas substâncias. Por conseguinte, o paciente irá manifestar inúmeros sinais clínicos com possível envolvimento neurológico ou somente sistêmico em decorrência do acúmulo das toxinas no sangue. O tratamento de eleição para o shunt portossistemico é a ligadura do vaso anômalo, à qual o anel constritor ameroide será o mais abordado nesse artigo.

Palavras chave: Anomalia vascular, constritor ameroide, desvio portossistêmico, encefalopatia hepática

\section{Congenital portosystemic shunt in dogs: review}

Abstract. Portosystemic shunt is a condition commonly seen in dogs. This disease is a vascular anomaly that makes communication between the systemic circulation and the portal circulation. Because of this anomalous vascular communication, portal blood from the drainage of organs such as the stomach, intestine, pancreas and spleen, passes directly into the systemic circulation, diverting from the liver that is responsible for detoxification of various substances. Therefore, the patient will manifest numerous clinical signs with possible neurological or systemic involvement only due to the accumulation of toxins in the blood. The treatment of choice for the portosystemic shunt is the ligation of the anomalous vessel, to which the ameroid constricting ring will be the most approached in this article.

Keywords: ameroid constrictor, hepatic encephalopathy, portosystemic shunt, vascular anomaly

\section{Desviación portosistémica congénita en perros: revisión}

Resumen. La desviación portosistémica o Shunt portosistémico es una afección comúnmente vista en perros. Esta enfermedad se trata de una anomalía vascular que hace comunicación entre la circulación sistémica y la circulación portal. Debido a esta comunicación vascular anómala, la sangre portal proveniente del drenaje de órganos como estómago, intestino, páncreas y bazo, pasa directamente a la circulación sistémica, desviando del hígado que es responsable de la detoxificación de diversas sustancias. Por lo tanto, el paciente manifestará numerosos signos clínicos con posible implicación 
neurológica o solamente sistémica como consecuencia de la acumulación de las toxinas en la sangre. El tratamiento de elección para el shunt portosistémico es la ligadura del vaso anómalo, a la cual el anillo constructor ameroide será el más abordado en ese artículo.

Palabras clave: anillo ameroide, anomalía vascular, desviación portosistémica, encefalopatía hepática, shunt portosistémico

\section{Introdução}

Dentre todos os órgãos internos do corpo, temos o fígado que se destaca no seu tamanho, sendo superior a todos os outros, constituindo $3 \%$ a $4 \%$ do peso corporal. O sangue que chega no fígado vem por meio de duas fontes. Uma delas é a veia porta à qual drena o sangue proveniente do trato digestório e representa cerca de $60 \%$ a $70 \%$ de todo o sangue que o fígado recebe e a outra fonte, é a artéria hepática responsável pelo restante do fluxo sanguíneo hepático. Pela veia hepática, o sangue sai do fígado, entrando na veia cava caudal (Cullen et al., 2006).

O fígado atua no metabolismo de lipídeos, carboidratos, proteínas e na desintoxicação de xenobióticos e metabólitos; no armazenamento de vitaminas, glicogênio e gordura, além da digestão de gordura; e, também, na imunorregulação (Webster, 2008). Outras atividades do fígado são: síntese de ureia, albumina, fibrinogênio e sais biliares; excreção de bilirrubina; armazenamento de sangue (Tobias \& Johnston, 2013). Por conseguinte, quando temos alguma alteração hepática, pode-se ter deficiência em uma ou mais das funções mencionadas (Webster, 2008).

O fígado do feto possui sua função limitada no processamento de produtos como: hormônios tróficos (intestinais e pancreáticos), nutrientes, toxinas derivadas do intestino e produtos de bactérias, provenientes da circulação portal, e como meio de proteção tem-se um grande vaso desviando da circulação hepática, o ducto venoso. A localização desse vaso fica no lado esquerdo do fígado, e o seu fechamento ocorre em torno de 3 a 10 dias após o nascimento. A oclusão do vaso ocorre após o fluxo venoso umbilical ser interrompido; e a contração da musculatura do ducto venoso, que ajuda no fechamento, ocorre em estimulação de compostos adrenérgicos ou tromboxano. A formação do desvio portossistêmico (DPS) ocorre quando esse ducto venoso permanece patente, ou então em decorrência da existência de outras comunicações congênitas (Weisse \& Berent, 2004).

Desvio portossistêmico ou shunt portossistêmico são vasos anômalos localizados entre a circulação sistêmica e a circulação portal. Sua origem pode ser congênita ou adquirida (Watson \& Bunch, 2015). Estes podem ser divididos em: desvios portossistêmicos intra-hepáticos congênitos (DPIH), que estão localizados no fígado e desvios portossistêmicos extra-hepáticos congênitos (DPSEC) ou adquiridos. A existência desses vasos anômalos permite que o sangue portal normal proveniente da drenagem do estomago, intestino, pâncreas, e baço, passe de maneira direta para a circulação sistêmica sem envolvimento do fígado (Fossum, 2014).

Conforme Watson \& Bunch (2015), os desvios portossistêmicos adquiridos ocorrem devido à hipertensão portal, que acontece em consequência de uma hepatite crônica e fibrose. Em decorrência da fibrose que faz com que os sinusoides se rompam, e que ocorre juntamente com obstrução, e também com o edema de hepatócitos, faz com que a pressão portal aumente até o ponto que ela ultrapassa a pressão da veia cava caudal, resultando na hipertensão portal. Em animais saudáveis, a pressão na veia porta é inferior àquela vista na veia cava caudal. Se esse aumento na pressão permanecer, formam-se os DPSs adquiridos; permitindo com que uma parcela do sangue portal não vá para o fígado.

\section{Sinais clínicos}

Os sinais clínicos no DPS congênito são comumente observados em torno de dois anos de idade, sendo esses sinais relacionados, principalmente, ao sistema nervoso. $\mathrm{O}$ animal pode apresentar andar compulsivo, pressionar a cabeça contra objetos, ataxia, letargia, torpor, sendo esses sinais clínicos os mais comumente observados em torno de 95\% dos casos (Jericó et al., 2015). Mehl et al. (2005), demonstraram que $82 \%$ dos cães possuíam sinais neurológicos, $76 \%$ com sinais gastrointestinais e 39\% sinais urinários. Segundo Dewey \& Costa (2016) em um estudo com 168 animais com desvio extrahepático, se obteve o resultado que somente um animal apresentava sinais de crises reativas, logo, não é frequente em animais com DPS. Sob outra perspectiva, 5\% dos casos, podem ter crises reativas no 
pós-operatório. Isso pode ocorrer devido a alterações nos níveis de serotonina no cérebro, que ocorrem de maneira abrupta. Além disso, são observados outros sinais como: diarreia, êmese, poliúria, polidipsia, e, ademais, hematúria pode ser vista devido à formação de cristais de biurato de amônio. Sinais que se assemelham com outras doenças neurológicas como: tetraparesias com deficiência de reação postural, amaurose e vestibulopatia podem ocorrer em animais com mais de cinco anos, podem aparecer (Jericó et al., 2015).

\section{Diagnóstico e exames complementares}

O diagnóstico é dificultado devido aos resultados dos exames laboratoriais de rotina, como hemograma completo, perfil bioquímico e urinálise serem inespecíficos. A função hepática está diminuída no caso de DPS, e alguns indicadores que nos auxiliam no diagnóstico são as concentrações de ácidos biliares séricos e amônia plasmática em jejum (Ruland et al., 2010). Também se faz a observação do vaso anômalo por meio de ultrassonografia, tomografia computadorizada (TC) ou portovenografia, ou ainda por cirurgia, observando o vaso macroscopicamente (Watson \& Bunch, 2015). As bactérias coliformes e anaeróbicas que estão presentes no intestino grosso formam a amônia. Quando temos a liberação da amônia no intestino, uma grande parte dela sofre conversão para ureia, nos hepatócitos no ciclo da ureia, por conseguinte, os aumentos nas concentrações de amônia no plasma são vistos em casos, como o DPS ocorre um defeito no ciclo da ureia causando incapacidade do fígado de transformar a amônia (Ruland et al., 2010). Além de a amônia ser convertida em ureia, ela também pode ser convertida em glutamina pelo mesmo clico. A substância neurotóxica mais importante do DPS é a amônia, devido ao fato de eventos metabólicos acontecerem em virtude do aumento da concentração deste, resultando em encefalopatia hepática em cães, ratos e humanos (Berent \& Tobias, 2013).

No diagnóstico de DPS, alguns testes têm sido usados. De acordo com a experiência e a preferência de cada médico veterinário é que vai ser feito a escolha do teste, além disso, o fator viabilidade e acessibilidade são importantes requisitos a serem levados em consideração na escolha. Dois testes estão dentre os mais usados no diagnóstico de DPS, sendo eles: concentração de amônia (AM) e concentração de ácidos biliares $(\mathrm{AB})$, os dois em jejum. Comumente, a concentração de $\mathrm{AB}$ em jejum está combinada com a concentração de AB no momento pós-prandial (Gerritzen-Bruning et al., 2006). Comparando os dois testes, a amônia possui uma maior especificidade do que os ácidos biliares para o diagnóstico de DPS, devido ao fato da colestase não interferir nos níveis de amônia. Por conseguinte, a amônia é ideal para o diagnóstico de DPS, ainda que seja feito numa população, com pacientes que tenham alguma doença hepática (Cullen et al., 2006).

Alterações nos níveis glicêmicos podem ser encontradas em algumas raças miniaturas, como o Yorkshire terrier, podendo alguns estar hipoglicêmicos. Comparando desvio portossistêmico extrahepáticos com intra-hepáticos, relata-se que a hipoglicemia é mais vista nos casos extra-hepáticos, em torno de $22 \%$, contra os $5 \%$ nos intrahepáticos. Essa diminuição nos níveis glicêmicos, pode ser em decorrência a uma diminuição das reservas de glicogênio, uma diminuição no catabolismo da insulina, ou então devido a uma endotoxemia (McConkey, 2000).

Conforme Watson \& Bunch (2015), alterações clinicopatológicas não específicas ocorrem em mais de $50 \%$ dos cães que são acometidos por DPS, dentre essas alterações temos: microcitose, hipoalbuminemia, elevações nas enzimas séricas ALT e FA, hipocolesterolemia e também uma baixa concentração na ureia. Conforme Watson \& Bunch (2015), quando se realiza o teste de amônia pósprandial, os valores sempre serão altos, mas quando feitos em jejum os valores podem não alterar.

Segundo Fossum (2014), a microhepatia é vista na maioria dos casos de DPS congênito, e ela varia de leve a acentuada. Esses animais dificilmente terão hepatomegalia ou fígado do tamanho normal. Outra alteração comumente vista, é um aumento do rim, em virtude de alterações vasculares. O exame de ultrassom é um dos métodos eleitos para diagnóstico de DPS. Entretanto, depende da experiência do operador. A formação de cálculos de urato de amônio é comum nesses pacientes, e se atribui a diminuição na produção de ureia e ao aumento na excreção renal de amônia, e esses fatores podem estar associados com infecções bacterianas no trato urinário. Estudos relatam que a formação de cálculos ocorre em 30\% dos animais com DPS (Berent \& Tobias, 2013). Conforme Fossum (2014) existem vários tipos de desvios extra-hepáticos descritos em caninos, dentre eles: da veia porta para a veia cava caudal, da veia porta para a veia ázigos, da veia gástrica esquerda, ou alguma de suas contribuintes, mesentérica 
caudal ou cranial, ou veia gastroduodenal conectando com a veia cava caudal, ou então pode ser algumas combinações feitas com veias anteriores.

\section{Tratamento}

Segundo Watson \& Bunch (2015) é preconizado que se faça um tratamento clínico, antes da cirurgia e por até oito semanas após, com o objetivo de estabilizar esses pacientes, enquanto o fígado ainda se recupera tanto em massa quanto no sentido vascular. Conforme Brainard \& Hofmeister (2012), os animais que apresentam DPS têm um risco maior para apresentar hipoglicemia, seja antes ou durante a anestesia. Em relação aos medicamentos anestésicos, esses animais tendem a prolongar a meia vida e alterar o metabolismo dos fármacos.

Em relação ao tratamento clínico, este pode ser realizado, agindo de maneira paliativa, controlando a insuficiência e encefalopatia hepática. Em relação aos medicamentos, antibióticos (p ex: metronidazol ou neomicina) podem ser usados em decorrência de esses atuarem na microbiota produtora de urease. A lactulose também pode ser utilizada, pois age no aumento da eliminação do conteúdo intestinal e também acidificando o lúmen intestinal, e com isso se tem a transformação de amônia em amônio (Brum et al., 2007).

De acordo com Watson \& Bunch (2015), as diretrizes sobre o manejo proteico desses animais mudaram, agora se sabe que esses animais necessitam de mais proteína do que aqueles animais que não possuem DPS. A restrição proteica pode causar uma desnutrição proteico-calórica, quando esta for realizada por longos períodos. Atualmente, se tem preconizado uma alimentação com proteínas digestíveis em pequenas quantidades, o que faz, desse modo, com que o trabalho do intestino delgado seja menor e isto termina por causar uma diminuição no metabolismo da glutamina. Entretanto, existem inúmeras técnicas no tratamento cirúrgico do DPS extra-hepático, dentre elas se tem a ligadura total ou então parcial do vaso anômalo. Essa ligadura pode ser feita com alguns produtos como: fio de seda, fita de celofane ou então com um anel "ameroide" a qual causa constrição (Jericó et al., 2015). Quando o DPS for adquirido, não se recomenda a cirurgia, logo a mesma só é realizada em casos de DPS congênito (Fossum, 2014). A técnica mais preconizada é aquela que oclui o vaso de maneira gradativa, fazendo com que o fígado assim possa se adaptar à nova pressão, pois se a oclusão for abrupta, pode-se ocasionar uma hipertensão portal aguda (Jericó et al., 2015). Caso, o vaso anômalo seja ligado de maneira parcial, pode-se ter mais predisposição para a ocorrência de sinais clínicos de maneira persistente ou ainda recorrente. Algumas estatísticas mostram que quando o vaso é ligado de maneira parcial se tem $32-50 \%$ dos casos com sinais clínicos recorrentes, e apenas $0-12 \%$ quando esse vaso for totalmente ligado (Besancon et al., 2004).

Na composição do ameroide temos: um anel de caseína desidratada e ao redor dela, temos um semicírculo metálico. O funcionamento se dá quando esse produto é colocado no vaso anômalo e essa caseína se reidrata com os líquidos provenientes da cavidade abdominal do animal, e com isso esse constritor vai sofrer uma expansão, ocluindo de maneira gradativa o vaso anômalo, dentro de 2 semanas a 3 meses (Jericó et al., 2015). A fim de avaliar o fluxo, em um estudo um vaso falso foi testado com o constritor ameroide, e os resultados obtidos foram que nos primeiros 4 dias uma rápida oclusão era notada, tendo visto que uma redução do fluxo acima de $50 \%$ já se via em torno de 48 horas, e acima de $80 \%$ em torno de quatro dias, e mais lentamente nos próximos dois meses (Besancon et al. (2004). Além de a parte interna ser constituída desse material higroscópico, ocorrendo então o inchaço desse produto num momento inicial, temos que a oclusão terminará em decorrência a uma fibrose ao redor do vaso anômalo (Fossum, 2014). A fim de se evitar a hipertensão portal, com a oclusão total do vaso anômalo após a colocação do ameroide, o ideal é que o cirurgião possua ao menos três tamanhos de constritores antes da cirurgia. Com isso, se evita também a oclusão parcial do vaso, quando se opta por um anel com tamanho superior (Jericó et al., 2015).

\section{Considerações finais}

Segundo esta pesquisa, existe a possibilidade de que ocorra a formação de novos desvios extrahepáticos, após a colocação do constritor ameroide, quando feita de maneira aguda ou então mais tardiamente. Com a ajuda da ultrassonografia, pode-se ter um número estimado acerca do diâmetro do vaso anômalo. Como recomendação, para o uso do anel constritor ameroide, não pode haver uma 
oclusão do vaso que ultrapasse $25 \%$ do seu tamanho, durante a colocação deste dispositivo. Atualmente, não há a venda do ameroide no Brasil, mas este pode ser importado, entrando em contato direto com o fabricante.

Portanto, é de suma importância que o médico veterinário saiba sobre o DPS, a sua fisiologia, sinais clínicos e os exames mais utilizados para o diagnóstico correto desse vaso anômalo o qual pode desencadear inúmeras alterações fisiológicas no animal. Ademais, a terapia medicamentosa é importante para estabilizar estes pacientes antes do procedimento cirúrgico, que é o método de solução para esses casos. É importante lembrar-se da maior ocorrência em animais de raça, sendo o Yorkshire terrier, a raça mais predisposta para essas anormalidades vasculares.

\section{Referências bibliográficas}

Berent, A. C. \& Tobias, K. M. (2013). Hepatic vascular anomalies. In K. M. Tobias \& S. A. Johnston (Eds.), Veterinary Surgery: Small Animal-E-BOOK: 2-Volume. St. Louis, USA: Elsevier Health Sciences.

Besancon, M. F., Kyles, A. E., Griffey, S. M. \& Gregory, C. R. (2004). Evaluation of the characteristics of venous occlusion after placement of an ameroid constrictor in dogs. Veterinary Surgery, 33(6):597-605.

Brainard, B. M. \& Hofmeister, E. H. (2012). Anesthesia principles and monitoring. Veterinary Surgery: Small Animal, 2248-290.

Brum, A. M., Champion, T., Zanatta, R., Costa, M. T. \& Canola, J. C. (2007). Utilização de probiótico e de lactulose no controle de hiperamonemia causada por desvio vascular portossistêmico congênito em um cão. Ciência Rural, 37(1):572-574.

Cullen, J. M., van den Ingh, T. S. G. A. M., Bunch, S. E., Rothuizen, J., Washabau, R. J. \& Desmet, V. J. (2006). Morphological classification of circulatory disorders of the canine and feline liver. WSAVA standards for clinical and histological diagnosis of canine and feline liver disease, 141-56.

Dewey, C. W. \& Costa, R. C. (2016). Practical guide to canine and feline neurology (3 Ed. ed.). New Delhi, India: Wiley Blackwell.

Fossum, T. W. (2014). Cirurgia de pequenos animais (4 ed. Vol. 1). São Paulo: Elsevier Brasil.

Gerritzen-Bruning, M. J., Van Den Ingh, T. S. G. A. M. \& Rothuizen, J. (2006). Diagnostic value of fasting plasma ammonia and bile acid concentrations in the identification of portosystemic shunting in dogs. Journal of Veterinary Internal Medicine, 20(1):13-19.

Jericó, M. M., Kogika, M. M. \& Andrade Neto, J. P. (2015). Tratado de medicina interna de cães e gatos. Rio de Janeiro, Brasil: Guanabara Koogan.

McConkey, S. (2000). Clinical pathology: portosystemic shunt in a Labrador retriever. The Canadian Veterinary Journal, 41(3):235-237.

Mehl, M. L., Kyles, A. E., Hardie, E. M., Kass, P. H., Adin, C. A., Flynn, A. K., . . Gregory, C. R. (2005). Evaluation of ameroid ring constrictors for treatment for single extrahepatic portosystemic shunts in dogs: 168 cases (1995-2001). Journal of the American Veterinary Medical Association, 226(12):2020-2030.

Ruland, K., Fischer, A. \& Hartmann, K. (2010). Sensitivity and specificity of fasting ammonia and serum bile acids in the diagnosis of portosystemic shunts in dogs and cats. Veterinary Clinical Pathology, 39(1):57-64.

Tobias, K. M. \& Johnston, S. A. (2013). Veterinary Surgery: Small Animal-E-BOOK: 2-Volume Set. St. Louis, USA: Elsevier Health Sciences.

Watson, P. J. \& Bunch, S. E. (2015). Doenças hepatobiliares no cão. In R. W. Nelson \& C. G. Couto (Eds.), Medicina Interna de Pequenos Animais (Vol. 1, pp. 556-557). Rio de Janeiro: Elsevier.

Webster, C. R. L. (2008). Cirrose e fibrose hepática. In L. P. Tilley \& F. W. K. Smith Junior. (Eds.), Consulta veterinária em 5 minutos: espécies canina e felina (pp. 226-228). São Paulo, Brasil: Manole. 
Weisse, C. \& Berent, A. C. (2004). Hepatic vascular anomalies. In S. Ettinger \& E. Feldman (Eds.), Tratado de medicina interna veterinária: doenças do cão e do gato (pp. 3998- 4023). Rio de Janeiro: Guanabara Koogan.

Recebido: 19 de junho, 2019.

Aprovado: 16 de julho, 2019

Publicado: 23 de setembro, 2019.

Licenciamento: Este artigo é publicado na modalidade Acesso Aberto sob a licença Creative Commons Atribuição 4.0 (CC-BY 4.0), a qual permite uso irrestrito, distribuição, reprodução em qualquer meio, desde que o autor e a fonte sejam devidamente creditados 